Journal of East Asian Cultures 2021/1: IX-X

DOI: $10.38144 /$ TKT.2021.1.1

http://orcid.org/0000-0002-8263-858X

birtalan.agnes@btk.elte.hu

\title{
Introduction \\ to the Project of the "East Asia" Thematic Group and its Peculiarities
}

\author{
ÁgNes Birtalan \\ (EDITOR)
}

Thematic Excellence Program: "Community Building: Family and Nation, Tradition and Innovation" supported by the National Research, Development and Innovation Fund offered several possibilities for the East Asia thematic research group. ${ }^{1}$ Under the aegis of the program, associates of the Institute of East Asian Studies had the opportunity to systematise thematically relevant research materials, compiled over many years, and to present them to the scholarly community. Colleagues who had not previously done research on the theme of family, nation and community building could analyse their materials from a new angle, i.e., based on their source corpuses and the project's criteria. This unified approach resulted in a collection of studies that is innovative, proving how the "intellectual capital" of diverse disciplines can be mobilized in targeted research and how its contents can be used as methodological material in education.

As the project leader, I set the goal to map the basic terminology in Eastern and Inner Asia in the first year, on which further research could be initiated and based. The main terminology in that year were the family, system of relations and rites of passages. Focusing on these central themes, the consequent essays sought to clarify variegated terminology and its usage, and there are articles discussing exactly the kinship "vocabulary" as Wuyingga's article based on her fieldwork investigates the dialectological problems of the specific terms of the Mongolian kinship system while Krisztina Nguyen studied the language teaching aspect of Korean kinship terminology. The former research of several colleagues was connected to the topic of traditional rites of passages and papers were completed prior to this project by Melinda Papp, Ramóna Fajkuszné Kovács, Judit Béres,

${ }^{1}$ The present project has been implemented with the support provided by the Ministry of Innovation and Technology of Hungary from the National Research, Development and Innovation Fund, financed under the ELTE TKP2020-IKA-06 funding scheme. 
and Aodunhu. Their contributions included in the volume present new results based on time-tested methodological approaches and their own collections from field research. Several scholars investigated the structure of the kinship system and its possible anomalies using historical sources as well, cf., Mónika Szegedi's and Alexa Péter's analysis of Tibetan materials, He Ruyi's interpretations of sources about the historical Uyghurs and Mátyás Balogh's discussion of several early Inner Asian peoples. As a new aspect of their ongoing research in religious studies, the central theme of the project was introduced in the interpretation of primary Buddhist sources, cf. the articles of Imre Hamar about Chinese, Beatrix Mecsi about Korean and Krisztina Teleki about Mongolian Buddhism. A popular and widely appealing theme is the research into the understanding of the notion of "family" in contemporary or pre-modern times, which explores the problems of affiliation in the families. Relevant to this theme are two studies looking into the social context in China by Zsombor Rajkai and the Chinese diaspora by the co-authors Eszter Knyihár and Gergely Salát. The situation of Japanese women in two significant periods of social transformation is examined by Ferenc Takó and the co-authors Yuko Umemura and Melinda Papp.

The above-reviewed volume, as a colourful yet unified collection of studies, is a worthy debut of the creative collective of the Institute of East Asian Studies. Unfortunately, the conference organised for 2020 with the participation of notable foreign experts had to be cancelled just one day before its start due to the pandemic situation. The practice of holding online conferences has only been introduced latterly. The papers of the volume do not entirely cover the material of the planned conference, as some papers delivered in 2020 are to appear in the volume of the next year for thematic considerations.

Let me also mention the workshop devoted to Mongolian themes held in September 2019 as an introduction to the project year. The apropos of the workshop was the Mongolian poet laureate Gombojawiin Mend-Ooyoo's visit to Hungary. In concert with the field research of the Department of Mongolian and Inner Asian Studies and the PhD-program in Mongolistics, the workshop topic was "The literary and visual anthropological representation of the Mongolian family". The programme and poster of the workshop are also appended to this volume.

As a new phase of the current project, we scrutinize the problem of identity (2021). Relying on broader resources of history, sociology, religion, culture and language, the project examines the crucial social and environmental questions of our day using the latest international research trends and methods, including a presentation of the utopian and dystopian possibilities of nation and family in the Eastern and Inner Asian region (2022). 